\title{
Design of the Multi-Channel Temperature Inspection System
}

\author{
Fei Quan ${ }^{1}$, Wei Wei ${ }^{2^{*}}$ \\ ${ }^{1}$ Shanxi Polytechnic Institute, Xianyang 712000, China \\ ${ }^{2}$ School of Computer Science and Engineering, Xi'an University of Technology, Xi'an 710048, China \\ *Corresponding authour: Wei Wei, taneo@126.com
}

Keywords: MSP430g2553; LM35; DS18B20; PT100; LCD12864

\begin{abstract}
The Multi-Channel Temperature inspection has been designed based on MSP430g2553 and temperature sensor. Through the MCU control peripheral circuit automatic inspection of 4 channel temperature is realized, and the results are displayed on LCD12864. How to design the temperature inspection including hardware and software is mainly described in detail. With the low power consumption of the MCU, the low power consumption of the system is realized in a simple structure which is of stable performance, economic and practical.
\end{abstract}

\section{Introduction}

In the modern industrial production, the accurate real-time collection of temperature has a high demand, at the same time in the face of the bad environment and the need for multiple detection situations, the staff of the site collection is not convenient. Therefore, there is a need to have a real-time multi-channel temperature acquisition device for accurate collection of the site temperature. Multi-channel temperature inspection system based on MSP430g2553 changes the traditional method of temperature measurement. It can collect the temperature of the field, and the simulated temperature value can be converted to the digital value displayed on the terminal LCD, through real-time detection of temperature, to achieve the purpose of monitoring of multi scene. This method can make the measurement accuracy, high stability and low power consumption [1-9].

\section{The Overall Design}

The system takes the MCU as the main controller of digital temperature inspection, with four different temperature sensors as the temperature information acquisition unit, the liquid crystal display and the driving element as the display unit. The basic block diagram of the system is shown in Fig.1.

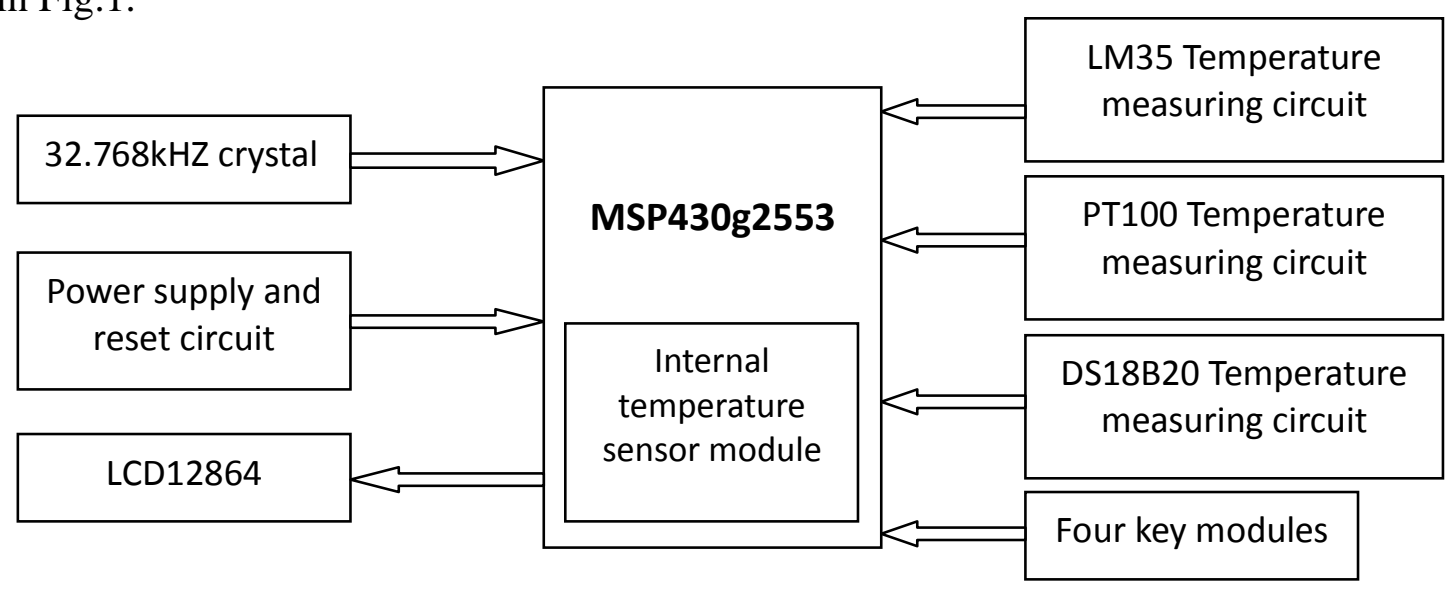


Fig. 1 General design of the system

\section{Hardware Design}

The multi-channel temperature inspection system has three kinds of temperature sensors, which are DS18B20, LM35 and PT100. MSP430g2553 single chip microcomputer system can use three kinds of temperature measurement module on the different temperature sensor and MCU internal temperature sensor to realize the temperature of the inspection. These three kinds of temperature measurement module through the MSP430g2553 microcontroller I/O port and ADC10 connection, to achieve real-time monitoring of temperature.

LM35 Temperature measuring circuit The voltage and temperature from the LM35 collected into a linear relationship, the formula is: Vout $=10.0 \mathrm{mV} /{ }^{\circ} \mathrm{C} * \mathrm{~T}^{\circ} \mathrm{C}, \mathrm{T}$ is the actual temperature value. The hardware circuit connected with MSP430g2553 is shown in Fig.2.

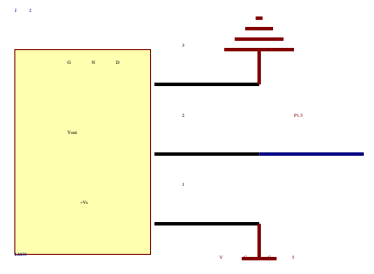

Fig. 2 LM35 hardware circuit connection diagram

DS18B20 temperature measurement circuit DS18B20 is characterized by the ability to directly output digital temperature, it is through the data line of DQ to communicate with MSP430g2553. It's hardware circuit connected with MSP430G2553 is shown in Fig.3.

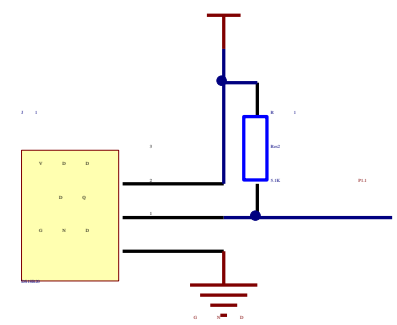

Fig. 3 DS18B20 hardware circuit connection diagram

PT100 Temperature measuring circuit PT100 is a platinum thermal resistance, the resistance will change with the change of temperature. In 0 degrees, the value of PT100 is $100 \mathrm{ohms,}$ and in 100 degrees, the value of PT100 is 138.5. The formula of the platinum resistance is:

$$
\mathrm{Rt}=\mathrm{R} 0(1+\mathrm{at}+\mathrm{bt} 2) \quad\left(0<\mathrm{t}<850^{\circ} \mathrm{C}\right)
$$


$\mathrm{Rt}$ is the resistance value of $\mathrm{T}$ degrees, $\mathrm{R} 0$ is the resistance value of 0 degrees celsius. The $\mathrm{A}$ and $\mathrm{B}$ coefficients in the formula are: $\mathrm{a}=3.90802 * 10-3^{\circ} \mathrm{C} ; \mathrm{b}=-5.802 * 10-7^{\circ} \mathrm{C}$ 。 The temperature measurement circuit of PT100 is shown in Fig.4.

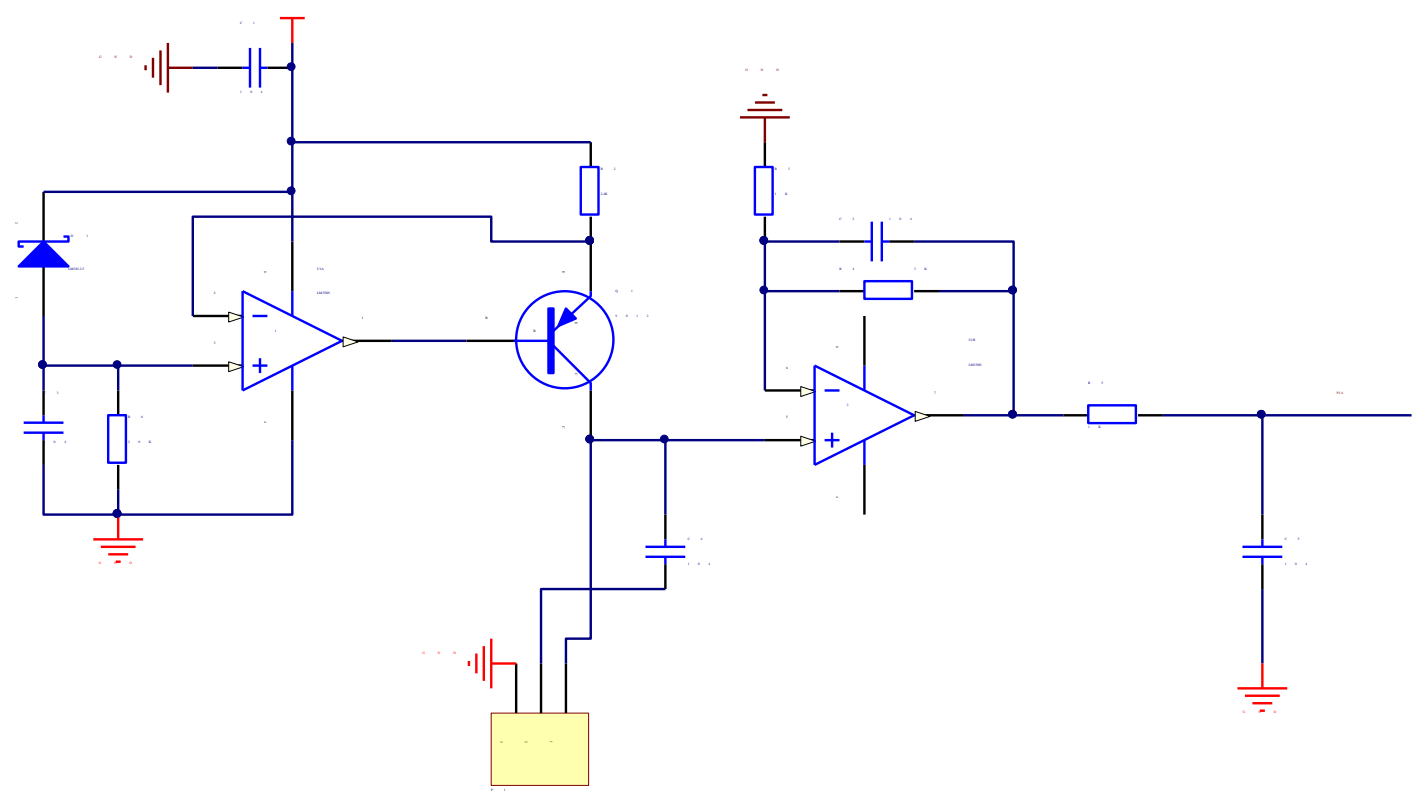

Fig. 4 PT100 hardware circuit connection diagram

Key control module The multi-channel temperature inspection system has four separate button which are respectively connected to the I/O ports of msp430g2553. The key of the system is used to realize the switching of the environmental temperature, which can display the temperature measurement value of the different temperature sensor. The function assignment value of the button is shown in Table 1:

Table 1 Button function table

\begin{tabular}{|c|c|c|}
\hline Button number & Corresponding I/O port & Function \\
\hline S1 & $\mathbf{P 2 . 1}$ & Display LM35 temperature \\
\hline S2 & $\mathbf{P 2 . 2}$ & Display DS18B20 temperature \\
\hline S3 & $\mathbf{P 2 . 3}$ & Display PT100 temperature \\
\hline S4 & $\mathbf{P 2 . 4}$ & $\begin{array}{c}\text { Display temperature of internal } \\
\text { temperature sensor }\end{array}$ \\
\hline
\end{tabular}

\section{Software Design}

Software design using CCS platform C language programming, the use of modular programming ideas, the different modules were programmed to facilitate the code of the transplant, call or modify. The software module is divided into 12864 basic modules: liquid crystal display module, LM35 temperature measurement module, DS18B20 temperature measurement module, PT100 temperature measurement module, internal temperature sensor temperature measurement module, button setting module.

\section{Summary}


The temperature acquisition and measurement of the system is practical, and it can be used to measure the temperature. At the same time, the system in the hardware design fully takes into account the scalability, in the I/O port more than several temperature sensors to achieve more points of temperature inspection.

\section{Acknowledgments}

We would like to thank the anonymous reviewers for their valuable comments. This work is supported by Beilin District 2012 High-tech Plan, Xi'an, China (No.GX1504) and supported by Xi'an science and technology project(CXY1440(6)) and Shaanxi Scientific research(2014k07-11).This job is also supported by Scientific Research Program Funded by Shaanxi Provincial Education Department (Program No.2013JK1139) and Supported by China Postdoctoral Science Foundation (No.2013M542370) and the Specialized Research Fund for the Doctoral Program of Higher Education of China (Grant No. 20136118120010). and supported by the Specialized Research Fund for the Doctoral Program of Higher Education of China Grant (No. 20136118120010)and supported by Shaanxi Province scientic and technological project (No.2013K06-09)and supported by National Natural Science Foundation of China (No.61305109 and No.61401324)and supported by 863 Item (No.2013AA014601).And this project is also supported by NSFC Grant (Program No. 11301414 and No.61272283 and No.61272283 and No.61472318).

\section{References}

[1]Wei Wei, Qi Yong. Information potential fields navigation in wireless Ad-Hoc sensor networks. Sensors, 2011, 11(5): 4794-4807.(UTW OS:000290947700019).

[2] Wei Wei, Xu Q, Wang L, et al. GI/Geom/1 queue based on communication model for mesh networks. International Journal of Communication Systems, 2014, 27(11): 3013-3029. (EI Accession number: 201436044545, 2014, 27(11): 3013-3029 UT WOS: 000345306300034).

[3] Wei Wei, Yang X L, Zhou B, et al. Combined energy minimization for image reconstruction from few views. Mathematical Problems in Engineering, 16(7): 2213-2223, 2012, 2012. (Article ID 154630, 15 pagesdoi:10.1155/2012/154630,SCI WOS:000310902600001, EI: 20124915745650).

[4] Wei Wei, Yang X L, Shen P Y, et al. Holes detection in anisotropic sensornets: Topological methods. International Journal of Distributed Sensor Networks, 21(9): 3216-3229, 2012, 2012. ( SCI WOS:000310624300001,EI: 20124915745593).

[5] Wei Wei, Qiang Y, Zhang J. A Bijection between Lattice-Valued Filters and Lattice-Valued Congruences in Residuated Lattices. Mathematical Problems in Engineering, 36(8): 4218-4229, 2013, 2013. (SCI:WOS:000322476900001, EI: 20133416634635).

[6] Wei Wei, Srivastava H M, Zhang Y, et al. A local fractional integral inequality on fractal space analogous to Anderson's inequality//Abstract and Applied Analysis. Hindawi Publishing Corporation, 46(8): 5218-5229, 2014, 2014.( Ariticle number: 797561, DOI: 10.1155/2014/797561, WOS:000339756400001, Year: 2014.

[7] Wei Wei, ZHANG Liang, GUO De-ke, SHEN Pei-yi, "Applications of information navigation method in wireless sensor networks", Journal on Communications, 2012. Vol. 33 No. Z2, Page: 146-152. (EI:20130515973180).

[8]Wang H, Wei W, Li Y, et al. Two-Degree-of-Freedom Congestion Control Strategy against Time Delay and Disturbance//Global Telecommunications Conference (GLOBECOM 2010), 2010 IEEE. IEEE, 2010: 1-5. (EI:20110613649206).

[9] Wei Wei.etc, Information fields navigation with piece-wise polynomial approximation for high-performance OFDM in WSNs 2013.7 Mathematical Problems in Engineering, ISSN:1024-123X 1-4 SCI:000316891600001,EI: 20131816284988. 